\title{
Structural system identification of thin web bridges by observability techniques considering shear deformation
}

\author{
Daniel Tomàs, Nexeo Solutions, Spain \\ Jose Antonio Lozano-Galant, Department of Civil Engineering, University of Castilla-La Mancha, Ciudad Real, Spain \\ Gonzalo Ramos, Department of Civil and Environmental Engineering, Universitat Politènica de Catalunya BarcelonaTech, Spain. \\ Jose Turmo, Department of Civil and Environmental Engineering, Universitat Politènica de Catalunya BarcelonaTech, Spain.
}

\begin{abstract}
Despite its importance in some structures, shear deformation is systematically neglected by most static structural system identification methods. This paper analyzes for the first time in the literature the effect of this deformation in the static inverse analysis of thin web bridges. This study is focused on the observability techniques. The most recent formulation found in the literature is based in the Euler-Bernoulli beam theory. This formulation is unable to identify correctly the characteristics of a structure (such as flexural stiffness) when shear deformation is not negligible. To solve this problem, the observability method is updated according to Timoshenko's beam theory. This formulation uses an algebraic method which combines a symbolical and a numerical application. Thus, the updated observability formulation is able to obtain not only the flexural stiffness but also the shear one. Besides this, a parametric equation of the estimates is obtained for the first time in the literature. Some examples of growing complexity are used to illustrate the validity of the new formulation.
\end{abstract}

KEYWORDS: Shear deformation, observability method, structural system identification, Timoshenko's beam.

\section{INTRODUCTION}

Damage in structures might produce changes in their mechanical properties. In order to quantify the magnitude of this damage Structural System Identification (SSI) might be used. This process is based on a subset of measured inputs and outputs (e.g. forces and/or displacements). Numerous papers about SSI have been written over the years. Sanayei et al. [1,2], Yan \& Golinval [3] or Liao et al. [4], proposed various methods to deal with different problems in SSI.

The subset of measured inputs can be obtained by non-destructive tests that measure the structural response under a certain load case. According to the load nature, these tests can be classified as dynamic $([5,6])$ or static ([7-9]). Focusing on static tests, Sanayei \& Onipede [10] presented an iterative optimization-based algorithm of the displacement equation error function for the parameter identification based on static test measurements. Banan et al. [11, 12] proposed an optimization method to estimate member constitutive properties of the Finite Element Model, FEM, from measured displacements under static loading. Sanayei et al. [13] used measured strains in a real bridge under static truck loads for FEM updating. However, in all these methods it is assumed that shear stiffness does not govern the problem and, therefore, it is not taken into account. This assumption is traditionally used in most SSI methods (see [14]).

Matrix methods of structural analysis are universally accepted in structural design. These methods enable a rapid and accurate analysis of complex structures under both static and dynamic conditions. However, when applying matrix methods, the system must be modeled as a set of simple, idealized elements interconnected at the nodes. Matrix SSI methods are based on simplified models of structures too. These include axial and flexural deformation. Therefore, neglecting this deformation can be assumed as a modeling error which is a simplifying assumption well justified in most of the cases. However, in some cases for both direct and inverse analysis, this modelling error can lead to unjustified rough results.

The assumption of neglecting shear deformation is explained by the fact that, for most structures, this effect is usually much smaller than the flexural one. Nevertheless, shear deformation might play an important role in some structures, such as deep beams. Eurocode EN 1992-1-1:2004 [15] defines deep beams as a beam for which the span is less than three times the overall section depth. ACI committee 318 [16], defines these elements based on two criteria: beams with clear spans equal to or less than four times the overall member depth or beams with concentrated loads within twice the member depth from the face of the support. In these structures, neglecting the shear deformation may affect adversely the stiffnesses estimated by SSI methods. Shear deformation also might be an important factor to be considered in some structures, for example, in high rise buildings (see [17, 18]). In this field, Li et al. [19] modeled slender structures such as high rise buildings and chimneys as cantilevers with both flexure and shear deformation. Recently, Ebrahimian \& Todorovska [20] presented a non-uniform Timoshenko beam model of a building, with piecewise constant properties along the height, along with an algorithm for structural system identification from earthquake records. In both papers, shear stiffness is clearly taken into account on damage detection. However, other authors neglect this phenomenon. Kang et al. [21] presented a system identification scheme in time domain to estimate stiffness and damping parameters of a structure using measured acceleration. Lei, Y. et al. [22] proposed an algorithm based on the extended Kalman estimator approach for the identification of structural parameters and unknown excitation of high rise shear-type buildings with partial acceleration responses. These papers are limited to identify the flexural stiffness and the story stiffness respectively.

According to Sahraei and Mohareb [23], shear deformation is traditionally neglected in thin-walled structures. Nevertheless, a number of studies discourage this assumption. Bhat and Oliveira [24] proposed the formulation of the shear coefficient of thinwalled prismatic beams. A formulation to incorporate the effects of shear deformation in thin-walled structures was proposed by 
Chen and Blandford [25] and Back and Will [26]. Shakourzadeh et al. [27] and Erkmen and Mohareb [28] studied the torsion analysis of thin-walled beams including the shear deformation effects. Van Phan and Mohareb [29] showed the importance of incorporating shear deformation effects when capturing predominantly torsional responses. Erkmen [30] studied the formulation for buckling analysis of thin-walled beams incorporating the shear deformation. Poul et al. [31] studied experimentally CFRP strengthened thin plated under shear loading. Chen et al. [32] analyzed the dynamic behavior of shear deformable sandwich beams. Hossain et al. [33] studied the impact shear resistance of double skin composite walls. Tong et al. [34] analyzed the behavior of plates subjected to combined bending and shear loading. Rassol and Singha [35] studied the nonlinear behavior of shear panels. Kim and Choi [36], Henriques et al. [37] and Sabouri-Ghomi et al. [38] studied the effects of shear deformation in composite beams. Analyses of the effect of shear in thin web bridges can be found in [39, 40].

The literature review shows that the effect of shear deformation is mainly based on the structural response at the element level. The studies about structural system identification including this phenomenon are restricted to dynamic excitations and the effects on static tests are not studied. This is the case of the observability techniques [41, 42]. As most of the static methods presented in the literature, it neglects the effect of the shear deformation into the structural system identification analysis. This paper analyzes how sensitive observability techniques are to shear deformation effects. Moreover, in order to take these into account, a new formulation including the shear effects in observability simulation is proposed. All numerical simulations are based on measurement error free data obtained from numerical analyses. Paper will focus on evaluating the modeling error linked with shear effects, being numerical and measurement errors on structural system identification by observability treated elsewhere [43, 44].

This article is organized as follows. In Section 2 the original observability method for structural system identification is briefly presented. This technique does not include the shear deformation. To illustrate the important role of this deformation in the identification of structures and to motivate the paper an example is analyzed. Section 3 introduces a new formulation to include the shear deformation into the observability analysis. To illustrate the application of this algorithm, a step by step example is presented. In addition, a numeric example is analyzed. Section 4 presents the application of the proposed algorithm for the structural system identification of a composite thin web bridge during its cantilevered construction. Finally, the conclusions obtained are displayed in Section 5.

\section{OBSERVABILITY ANALYSIS WITHOUT SHEAR DEFORMATION}

The stiffness matrix method is the most common implementation of the Finite Element Method (FEM) for structural analysis. The implementation of this method requires that the structure is modeled as a set of simple, idealized elements interconnected at the nodes. The material and stiffness properties of these elements are then compiled into a single matrix equation which governs the behavior of the entire idealized structure. In 2D, the traditional stiffness matrix $[\mathrm{K}]$ for a six degrees of freedom (two deflections ( $\mathrm{u}$ and v) and one rotation (w) at the initial and final beam element nodes), beam element of length $L$ and constant cross-section is:

$$
[K]=\left|\begin{array}{cccccc}
\frac{E A}{L} & 0 & 0 & -\frac{E A}{L} & 0 & 0 \\
0 & \frac{12 E I}{L^{3}} & \frac{6 E I}{L^{2}} & 0 & -\frac{12 E I}{L^{3}} & \frac{6 E I}{L^{2}} \\
0 & \frac{6 E I}{L^{2}} & \frac{4 E I}{L} & 0 & -\frac{6 E I}{L^{2}} & \frac{2 E I}{L} \\
-\frac{E A}{L} & 0 & 0 & \frac{E A}{L} & 0 & 0 \\
0 & -\frac{12 E I}{L^{3}} & -\frac{6 E I}{L^{2}} & 0 & \frac{12 E I}{L^{3}} & -\frac{6 E I}{L^{2}} \\
0 & \frac{6 E I}{L^{2}} & \frac{2 E I}{L} & 0 & -\frac{6 E I}{L^{2}} & \frac{4 E I}{L}
\end{array}\right|
$$

where $E, A$ and $I$ are Young modulus, area and inertia respectively.

\subsection{Direct analysis of the stiffness matrix method}

In static structural analysis, a statement of the equilibrium conditions together with strength of materials theory leads to a relation between forces and displacements that has the form of a system of equations:

$[K] \cdot\{\delta\}=\{f\}$,

where $\{\delta\}$ and $\{f\}$ are the vectors of displacements and forces, respectively, in which the stiffness matrix is a singular matrix that leads to a system with infinite solutions For a more detailed explanation about the unicity of the solution of a system of polynomial equations the reader is addressed to $[50,51]$.

\subsection{Inverse analysis of the stiffness matrix method by observability techniques}

As it was mentioned in the introduction, in actual structures, unknown parameters, such as the flexural stiffness $E I_{j}$ or axial stiffness $E A_{j}$ of element $j$, may appear into the matrix $[K]$ due to damage or other uncertainties. These unknowns might be due to damage (e.g. by material degradation, such as carbonation or corrosion, or accidental actions, such as fires or impacts) or other uncertainties (e.g. lack of knowledge about the mechanical properties of the material). If the external forces applied to the structure in a non-destructive test are known and some displacements are measured, the observability method can be applied into the SSI to 
found the values of these unknown parameters. Taking Eq. (2) where the matrix $[K]$ is partially unknown and with the aim of determining the value of the unknown stiffnesses $\left(E A_{j}\right.$ and $\left.E I_{j}\right)$ a modified system of equations can be rewritten as:

$$
\left[K^{*}\right] \cdot\left\{\delta^{*}\right\}=\{f\}
$$

in which the products of unknowns are located in the modified vector of displacements $\left\{\delta^{*}\right\}$ and the modified stiffness matrix $\left[K^{*}\right]$ is a matrix of known coefficients with different dimensions than the initial stiffness matrix $[K]$. The new system leads to a nonlinear problem due to the fact that unknown parameters, such as axial stiffness $E A_{j}$ and flexural stiffness $E I_{j}$ of the cross section are multiplied by the unknown horizontal displacements $\left(u_{i}\right)$, vertical displacements $\left(v_{i}\right)$ and rotations at the $i t h$ node $\left(w_{i}\right)$ of vector $\left\{\delta^{*}\right\}$. This fact implies that non-linear products of variables, such as $E A_{j} u_{i}, E A_{j} v_{i}, E I_{j} u_{i}, E I_{j} v_{i}$ and $E I_{j} w_{i}$ might appear, leading to a polynomial system of equations. These kinds of problems usually appear in science and engineering fields, (see [46, 47]). Depending on the known information, the unknown variables of vector $\left\{\delta^{*}\right\}$ may be the non-linear products presented above, as well as other factors of single variables, such as $E I_{j}, E A_{j}$ or nodal deflections and rotations.

Once the boundary conditions and the applied forces in the nodes have been defined, an inverse analysis can be performed to identify the unknown parameters. To do so, some deflections and rotations are measured in a known static test. This known information is clustered in a subset $\delta_{1}^{*}$ of $\left\{\delta^{*}\right\}$ and a subset $f_{1}$ of $\{f\}$, respectively. In this way, the remaining subset $\delta_{0}^{*}$ of $\left\{\delta^{*}\right\}$ and $f_{0}$ of $\{f\}$ are unknown. Hence, (3) can be written as follows:

$$
\left[K^{*}\right] \cdot\left\{\delta^{*}\right\}=\left[\begin{array}{cc}
K_{00}^{*} & K_{01}^{*} \\
K_{10}^{*} & K_{11}^{*}
\end{array}\right] \cdot\left\{\begin{array}{l}
\delta_{0}^{*} \\
\delta_{1}^{*}
\end{array}\right\}=\left\{\begin{array}{l}
f_{0} \\
f_{1}
\end{array}\right\}=\{f\}
$$

To join the unknowns, this system can be rewritten in the equivalent form:

$$
[B] \cdot\{z\}=\left[\begin{array}{cc}
K_{10}^{*} & 0 \\
K_{00}^{*} & -I
\end{array}\right] \cdot\left\{\begin{array}{l}
\delta_{0}^{*} \\
f_{0}
\end{array}\right\}=\left\{\begin{array}{c}
f_{1}-K_{11}^{*} \delta_{1}^{*} \\
-K_{01}^{*} \delta_{1}^{*}
\end{array}\right\}=\{D\},
$$

where 0 and $I$ are the null and the identity matrices, respectively. In this system, the vector of unknowns, $\{z\}$, appears on the lefthand side and the vector of observations, $\{D\}$, on the right-hand side. Both vectors are related by a matrix of known coefficients [ $B]$.

To evaluate if the system has a solution, it is sufficient to calculate the null space $[V]$ of $[B]$ and check that $[V]^{T}\{D\}=0$. Note that the null space can be easily obtained from the singular value decomposition by using computer programs. If this holds, the system is compatible; otherwise, it has no solution [48]. The general solution (the set of all solutions) has the structure:

$$
\left\{\begin{array}{l}
\delta_{0}^{*} \\
f_{0}
\end{array}\right\}=\left\{\begin{array}{l}
\delta_{00}^{*} \\
f_{00}
\end{array}\right\}+[V] \cdot\{\rho\}
$$

where $\left\{\begin{array}{l}\delta_{00}^{*} \\ f_{00}\end{array}\right\}$ is a particular solution of the system. This particular solution can be obtained by using two different subroutines in Matlab [49]. The first one is the backslash function $(\backslash$ that is able to find the parametric equations of the systems and therefore the symbolic and the numerical solution. The other one is the Moore-Penrose pseudoinverse function (pinv) with which only the numerical solution can be found.

$[V]\{\rho\}$ is the set of all solutions of the associated homogeneous system of equations (a linear space of solutions, where the column of $[V]$ is a basis and the elements of the vector $\{\rho\}$ are arbitrary real values that represent the coefficients of all possible linear combinations). It is interesting to note that a variable has unique solution not only when matrix [ $V]$ has zero dimensions (it does not exist), but when the associated row in matrix $[V]$ is null. Thus, examination of matrix $[V]$ and identification of its null rows lead to identification of the observable variables (defined as the subset of variables with a unique solution) of vector $\{z\}$. By solving system (5), the numerical value of the observed parameters obtained by the particular solution of Equation (6) can be easily found. To obtain these numerical values of the observable parameters it is necessary to combine the symbolical and the numerical analysis as proposed by Nogal et al. [50].

A recursive process is used to minimize the number of required deflections in measurement sets to obtain the unknown parameters. This process takes advantage of the connectivity of the beams in the stiffness matrix that is included in the partitioned matrices of $\left[K^{*}\right]$ in system (5). In this way, when in the initial observability analysis any deflection, force or structural parameter is observed, this information might help to observe new parameters in the adjacent elements through the recursive process. In this analysis, the observed information is successively introduced as input data in the observability analysis. A more detailed explanation of this process can be found in [51].A new approach for the numerical solution of the system based on compatibility equations can be found in [52].

In order to show the role of the shear deformation in the SSI by observability techniques an illustrative example is numerically 
analyzed in the following section.

\subsection{Example 1: Effect of the shear deformation on beams}

To motivate this paper, a sensitivity analysis is performed to evaluate the effect of the shear deformation on the SSI of a simply supported concrete beam loaded with a concentrated load $Q$ at mid span (Fig. 1). Young's modulus is $E=27000 M P a$ and Poisson's ratio is $v=0.25$. The beam has a rectangular cross section $1 \mathrm{~m}$ deep $(h=1 \mathrm{~m})$ and $0.1 \mathrm{~m}$ wide $(b=0.1 \mathrm{~m})$, so its area is $A=0.1$ $\mathrm{m}^{2}$ and its inertia is $I=0.00833 \mathrm{~m}^{4}$. Besides this, and according to [53], the shear coefficient $(k)$ for rectangular cross-sections when width/depth ratio approaches zero is $k=5 / 6$. With this shear factor, the shear area $\left(A_{v}\right)$ is equal to $A_{v}=0.08333 m^{2}$.

In this simple example with only one unknown (I), inertia can be inferred from the deflected shape of the beam, that will include shear deformation. If this is neglected, Euler-Bernoulli theory will be used to obtain the estimated inertia $(\hat{I})$. Otherwise, Timoshenko's theory will be used to obtain the allegedly real inertia $(\bar{I})$

Fig. 1.A shows the increment of deflections due to shear and bending deformability for a particular example of a simply supported beam with a concentrate load $\mathrm{Q}$ at mid span in terms of $\mathrm{L} / \mathrm{h}$. The shear deflection varies linearly with $\mathrm{L}$ while the bending deformability depends on $\mathrm{L}^{3}$. Fig. 1.B shows the error on estimating the inertia (in percentage) using Euler-Bernoulli theory in beams with non-negligible shear deformation. This error is presented as a function of the span-to-depth ratio. Span length is the variable used to modify the $L / h$ ratio. This variable is changed from 2 to $20 \mathrm{~m}$. The lowest value of $2 \mathrm{~m}$ is selected to avoid a very deep beam where Saint-Venant's principle cannot be applied at all. The limits of $2 \%$ and $5 \%$ are highlighted in this figure. In addition, the ratio limits for deep beams proposed by the Eurocode EN [15] and by the ACI Committee 318 [16] are also indicated. As expected, this graph illustrates how the slender the beam, the lower the effect of the shear deformation is.
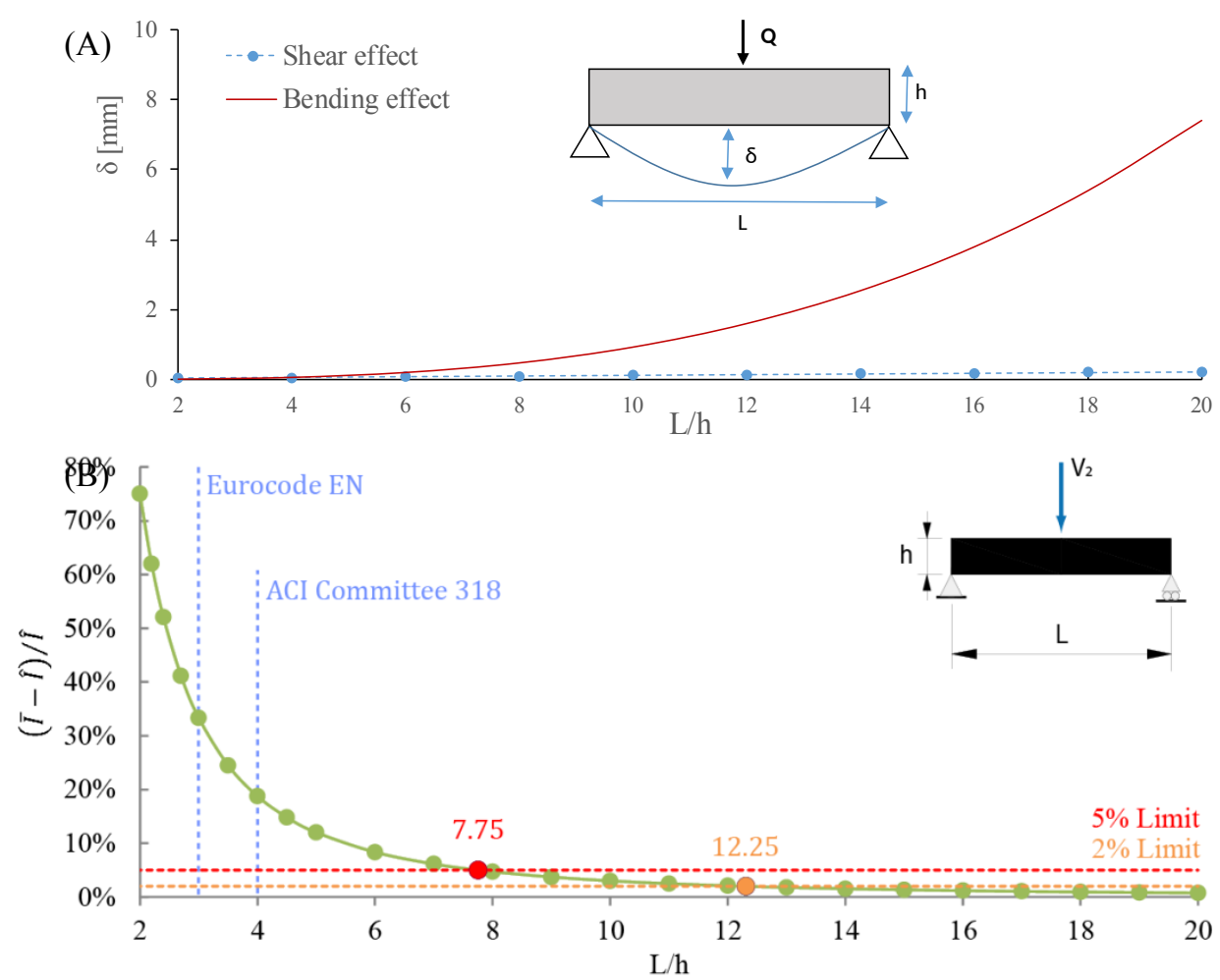

Figure 1: Example 1.(A) Increment of deflection at mid span due to bending and shear for a specific beam. (B) Percentage of inertia estimation error depending on the span-to-depth ratio when shear deformation is not modelled. Deep beam ratio limit for EN and ACI highlighted.

In Fig. 1.B, $5 \%$ error is achieved when the span-to-depth ratio is greater than 7.75 . In the same way, $2 \%$ error corresponds to a beam span-to-depth ratio of 12.25. If a uniform distributed load $(q)$ along the beam is used instead of the concentrated load $(Q)$, the span-to-depth ratios associated with a $2 \%$ and $5 \%$ error are reduced to 6.9 and 10.95 , respectively. This analysis shows how shear deformation effects are only negligible for SSI of beams with high span-to-deep ratios.

\subsection{Example 2: Effect of the shear deformation on SSI by observability techniques}

To illustrate the problems of neglecting the shear deformation into the observability analysis, the simply supported beam described in Figure 2.A is analyzed. This beam has a length of 6 meters and it is simulated with a Finite Element Model composed by 6 beam elements and 7 nodes. The length of each of these elements is defined as 1 meter. The mechanical and geometrical properties of the analyzed example correspond with the beam presented in the preceding section, giving a length to depth ratio of 6 . The boundary conditions of the structure are horizontal and vertical displacements restricted in node 1 and vertical displacement restricted in node $7\left(u_{1}=v_{1}=v_{7}=0\right)$ and the only external force applied is a concentrated vertical force in node 3: $V_{3}=-100 \mathrm{kN}$.

The beam is analyzed with Midas/Civil software [45] with and without shear deformation. The deformed shapes of the beam including only bending deformation, corresponding to $v_{i}$ and $w_{i}$, or bending and shear deformation, $v s_{i}$ and $w s_{i}$, are presented in 
Figure 2.B, being $i$ the node number.

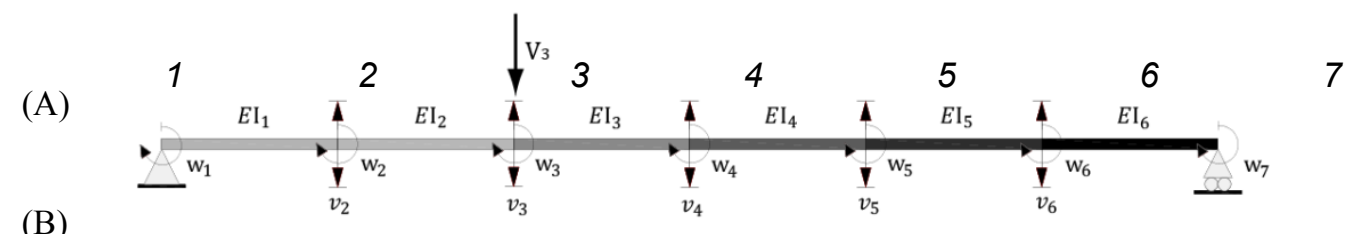

(B)

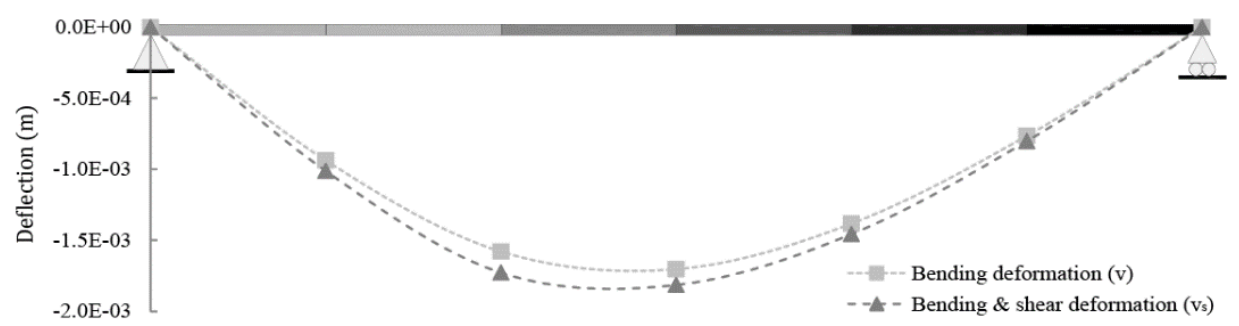

Figure 2: Example 1. (A) Structure with applied load including vertical deflections, $v$, and rotations, w. Nodes in italics. (B) Deformed shape with bending deformation, $v$, or with bending and shear deformation, vs.

The deformed shapes will be used to estimate the value of all the flexural stiffnesses $\left(E I_{1}\right.$ to $\left.E I_{6}\right)$. In order to do so, the measurement of at least 6 deflections or rotations that represent the flexural behavior of the beam are required. The selection of these measurements was analyzed by the observability trees method proposed in [41]. From the application of this procedure, it can be concluded that measuring the five vertical deflections of the inner nodes $\left(v_{2}\right.$ to $\left.v_{6}\right)$, and the rotation of node $3\left(w_{3}\right)$ will enable the estimation of all the unknown stiffnesses, or in other words, will provide full observability of the system.

Once the measurement set is selected, the observability method proposed by Nogal et al. [50] is applied using the two following measurement sets: (1) Measurements without shear deformation, which is $v_{2}$ to $v_{6}$ and $w_{1}$. (2) Measurement with shear deformation, that is $v s_{2}$ to $v s_{6}$ and $w s_{1}$. The observable flexural stiffnesses $(\hat{E I})$ obtained throughout the recursive process for both measurement sets are presented in Figure 3 as percentage deviations.

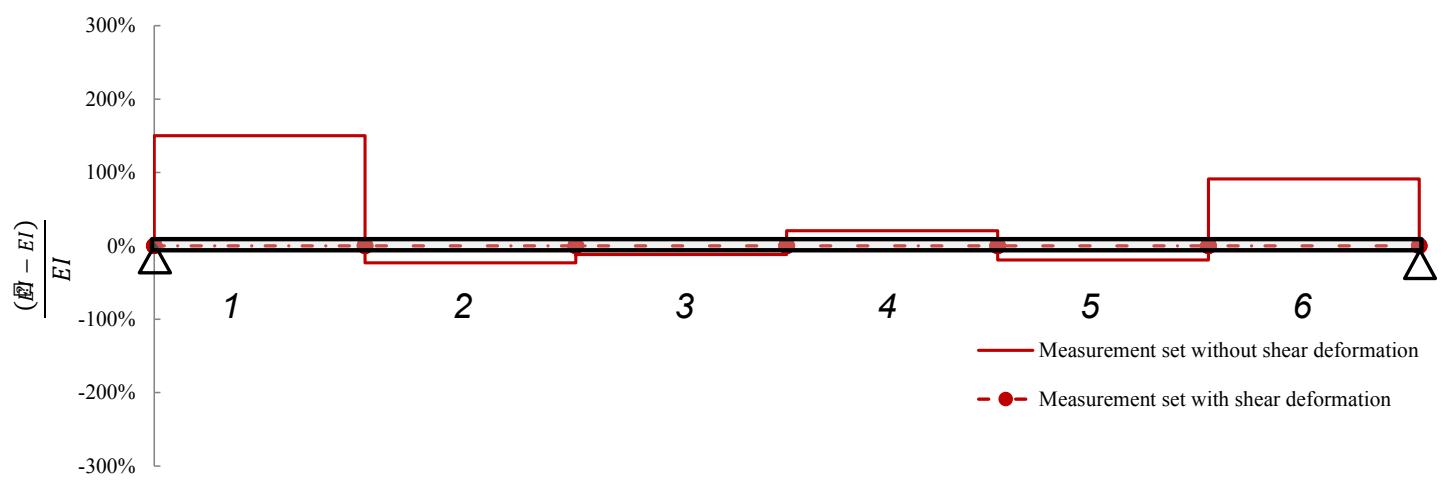

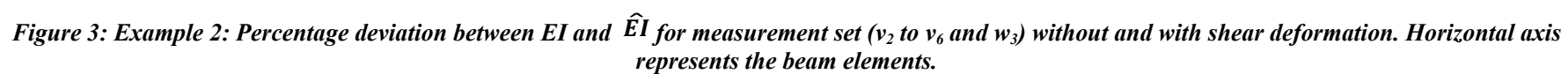

The analysis of Figure 3 shows no deviations in $\hat{E I}$ when measurements without shear deformation are considered. Nevertheless, this is not the case when the shear deformation is included, as large errors are found in $\hat{E I}$. For example errors of $149.9 \%$ are found in the first beam element. This disproportionate error is explained by the fact that the used model does not consider shear effects. In fact, shear deformation is considered as a modeling error [13] into the observability analysis.

The obtained results show that the role of shear deformation cannot be always neglected in the structural system identification by observability techniques. Thus, it can be concluded that a reformulation of the observability algorithm is required to include the effect of the shear deformation. This new algorithm is presented in the following section.

\section{INTRODUCING SHEAR DEFORMATION INTO THE SSI BY OBSERVABILITY ANALYSIS}

With the aim of including shear deformation into the SSI by observability analysis a new formulation of the stiffness matrix method is implemented. This formulation is based on the Timoshenko's beam theory. Then, an illustrative step by step example is presented to explain the differences with the traditional observability method presented in the preceding section and in the literature. 


\subsection{Shear formulation}

The stiffness matrix $[K]$ for a beam element with uniform cross section with 3 degrees of freedom per node $\left(u_{i}, v_{i}\right.$ and $\left.w_{i}\right)$ is of order $6 \times 6$. The forces developed in the beam member when a unitary displacement is imposed along each degree of freedom holding all other displacements equal to zero leads to the finding of all the single elements of this stiffness matrix. According to the existing literature [54], the stiffness matrix $[K s]$ for a 2D analysis of a six degrees of freedom (two deflections ( $u$ and $v)$ and one rotation (w) at the initial and final beam element nodes), beam element including the shear deformation is:

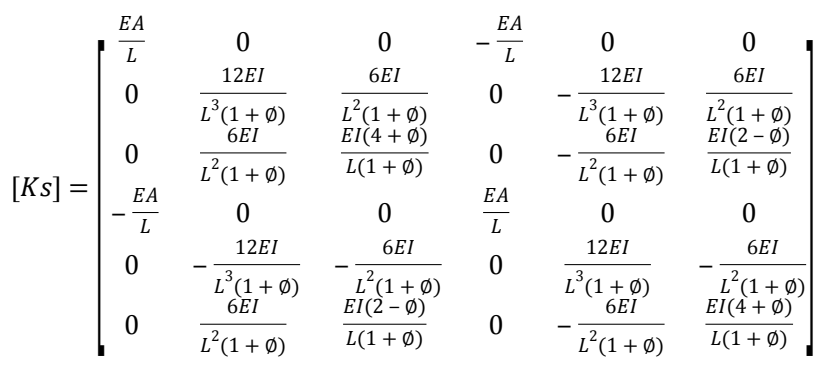

A comparison with the traditional stiffness matrix presented in Equation (1) shows that differences appear in some terms in the form of a new coefficient. This coefficient is known as the shear parameter $(\varnothing)$ and can be expressed as:

$$
\emptyset=\frac{12 E I}{G A_{v} L^{2}}
$$

where $G$ is the shear modulus and can be calculated as:

$$
G=\frac{E}{2(1+v)}
$$

As it can be appreciated in Equation (7), the shear stiffness matrix has the same coefficients $\left(\frac{E A}{L}\right)$ for the axial stiffness as the traditional stiffness matrix. This is because the shear and flexural stiffnesses are uncoupled from the axial stiffness. However, for the rest of the non-null elements of the matrix, shear and flexural stiffnesses are coupled.

When the beam is subjected to shear forces and associated moments, the vertical deflection is $v=v_{b}+v_{s}$ (where $v_{b}$ is the deflection due to the flexural strains and $v_{s}$ is the additional deflection due to shear strains). Analysis of Equation (7) shows that the shear parameter $\varnothing$ appears in both the numerator and the denominator of the matrix terms. Its location complicates significantly its estimation. In fact, the resulting system cannot be easily solved with observability techniques. To solve this problem a change of variable from $\varnothing$ to $Q$ is proposed. This change simplifies the mathematical resolution of the system and enables the application of observability techniques as the new unknown parameter appears uniquely in the numerator of the matrix terms. The definition of parameter Q is as follows:"

$$
Q=\frac{\emptyset}{1+\emptyset}
$$

where $Q$ is the so called observability shear parameter of the stiffness matrix [Ks]. It is to highlight that the value of this ranges from 0 to 1 . After introducing this change of variable, the stiffness matrix presented in Equation (7) is updated to the following form:

$[K S]=\left[\begin{array}{cccccc}\frac{E A}{L} & 0 & 0 & -\frac{E A}{L} & 0 & 0 \\ 0 & \frac{12 E I}{L^{3}}-\frac{12 E I Q}{L^{3}} & \frac{6 E I}{L^{2}}-\frac{6 E I Q}{L^{2}} & 0 & -\frac{12 E I}{L^{3}}+\frac{12 E I Q}{L^{3}} & \frac{6 E I}{L^{2}}-\frac{6 E I Q}{L^{2}} \\ 0 & \frac{6 E I}{L^{2}}-\frac{6 E I Q}{L^{2}} & \frac{4 E I}{L}-\frac{3 E I Q}{L} & 0 & -\frac{6 E I}{L^{2}}+\frac{6 E I Q}{L^{2}} & \frac{2 E I}{L}-\frac{3 E I Q}{L} \\ -\frac{E A}{L} & 0 & 0 & \frac{E A}{L} & 0 & 0 \\ 0 & -\frac{12 E I}{L^{3}}+\frac{12 E I Q}{L^{3}} & -\frac{6 E I}{L^{2}}+\frac{6 E I Q}{L^{2}} & 0 & \frac{12 E I}{L^{3}}-\frac{12 E I Q}{L^{3}} & -\frac{6 E I}{L^{2}}+\frac{6 E I Q}{L^{2}} \\ 0 & \frac{6 E I}{L^{2}}-\frac{6 E I Q}{L^{2}} & \frac{2 E I}{L}-\frac{3 E I Q}{L} & 0 & -\frac{6 E I}{L^{2}}+\frac{6 E I Q}{L^{2}} & \frac{4 E I}{L}-\frac{3 E I Q}{L}\end{array} \mid\right.$

Note that the shear parameter $\varnothing$ appearing in Equation (7) is replaced by the observability shear parameter $Q$ in Equation (11). Thus, new nonlinear products of variables appear.

When this new formulation of the stiffness matrix is applied to the observability method a new observability variable has to be calculated in addition to the axial $(E A)$ and flexural $(E I)$ stiffness. This new variable is named observability shear stiffness $(E I Q)$ and it is related to the shear deflections and to the shear parameter $\emptyset$. Besides this, the observability shear parameter can be expressed in terms of $\left(E A_{v}\right)$ with the following equation:

$E A v=\frac{-24 E I(v+1)(Q-1)}{L^{2} Q}$ 
The fact of having new unknowns $(E I Q)$ leads to the necessity of adding additional measurements to be able to identify them. Thus, it is necessary to use a new measurement set to be able to obtain all the observable variables $(E A, E I$ and $E I Q)$ of the structure.

\subsection{Step by step application of observability to shear stiffness}

The proposed algorithm is applied into a step by step example. In this example, a simple structure will be analyzed to illustrate the whole process necessary to obtain the unknown stiffnesses. This example is not focused on the numerical approach but on a symbolical one. In fact, for the first time in the literature, the parametric equations of the estimations are presented. Therefore, the numerical values could be easily obtained if necessary.

The structure considered in this academic example is a simply supported beam composed by 2 beam elements of a length $L$ and 3 nodes as shown in Figure 4. There are two concentrated forces applied at node $2\left(U_{2}\right.$ and $\left.V_{2}\right)$ and six non-restrained displacements and rotations: two horizontal displacements in nodes 2 and $3\left(u_{2}\right.$ and $\left.u_{3}\right)$, one vertical displacement in node $2\left(v_{2}\right)$ and three rotations in nodes 1,2 and $3\left(w_{1}, w_{2}\right.$ and $\left.w_{3}\right)$. Finally, the unknowns in this structure are: two axial stiffnesses $\left(E A_{1}\right.$ and $\left.E A_{2}\right)$, one flexural stiffness $(E I)$ and one observability shear stiffness $(E I Q)$. The fact that elements with the same bending stiffness have different axial stiffness does not have any physical meaning. However, this choice is purposely done for the sake of a better representation of the step-by-step example. The set of measurements needed to obtain the unknown parameters has to be composed at least by the same number of unknown stiffnesses (this is, 4 measurements). The selected measurements are $w_{1}$, $u_{2}$ ,$v_{2}$ and $w_{2}$.

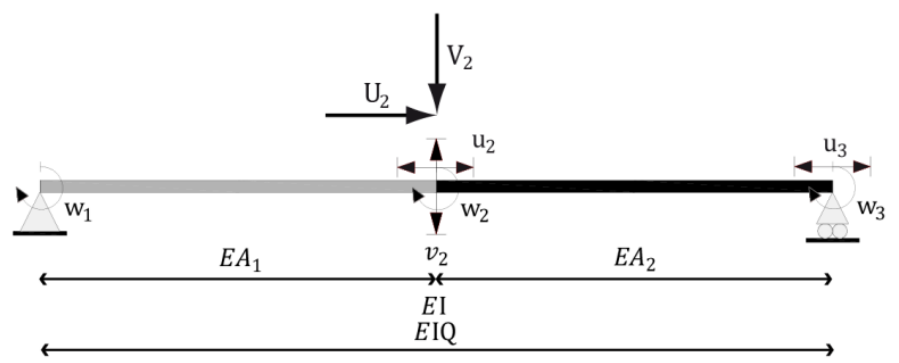

Figure 4: Two element simply supported beam model including unknown stiffnesses, load cases and measurement set.

In the structure presented in Figure 4, the equation that links displacements $\left\{\delta_{1}\right\}$ and forces $\{f\}$ through the stiffness matrix $\left[K s_{1}\right]$ is as follows:

$$
\left[K s_{1}\right] \cdot\left\{\delta_{1}\right\}=\{f\}
$$

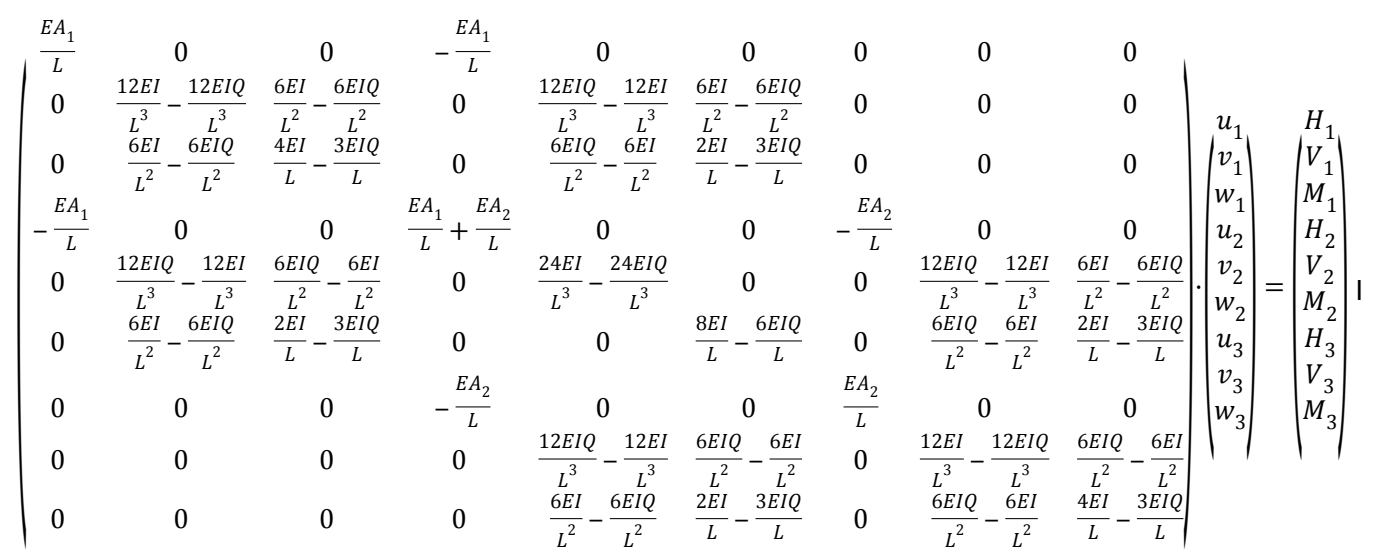

In matrix $\left[K s_{1}\right]$, the flexural and the observability shear stiffnesses are mixed in columns $2,3,5,6,7$ and 8 . To separate these unknowns it is necessary to uncouple these terms. To do this, the system of equations in Equation (13) is rewritten, modifying the stiffness matrix by separating the terms made of several summands. After this step, a new matrix $\left[K s_{2}\right]$, where each parameter corresponds to a single column is obtained. The new system is as follows:

$$
\left[K s_{2}\right] \cdot\left\{\delta_{2}\right\}=\{f\}
$$




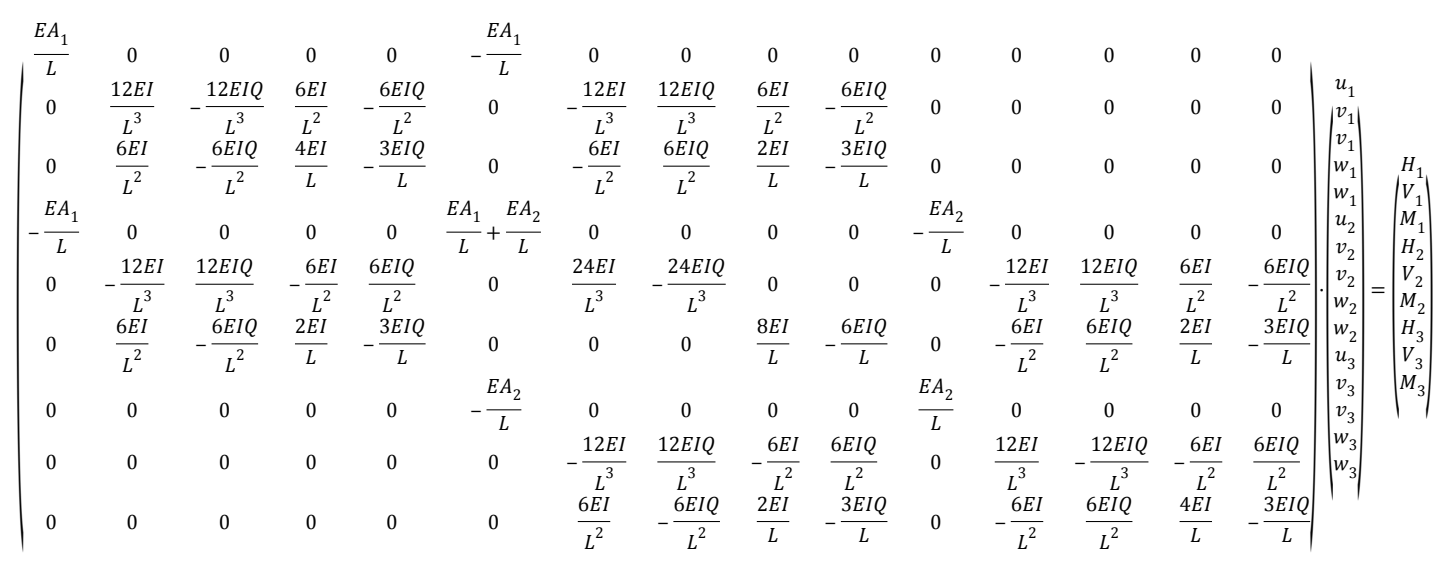

As all the stiffnesses are unknown, they have been moved to the vector $\delta_{2}^{*}$, as it is shown in Equation (15). This vector is composed of non-linear terms including the stiffnesses $\left(E A_{1}, E A_{2}\right.$, EI and EIQ) coupled with nodal deflections $\left(u_{i}, v_{i}, w_{i}\right)$. This rearrangement transforms matrix $\left[K s_{2}^{*}\right]$ to a matrix of known coefficients. Equation (15) shows the modified system:

$$
\begin{aligned}
& {\left[K S_{2}^{*}\right] \cdot\left\{\delta_{2}^{*}\right\}=\{f\}} \\
& \begin{array}{cccccccccccccccc}
\frac{1}{L} & 0 & 0 & 0 & 0 & -\frac{1}{L} & 0 & 0 & 0 & 0 & 0 & 0 & 0 & 0 & 0 & 0 \\
0 & \frac{12}{L^{3}} & -\frac{12}{L^{3}} & \frac{6}{L^{2}} & -\frac{6}{L^{2}} & 0 & 0 & -\frac{12}{L^{3}} & \frac{12}{L^{3}} & \frac{6}{L^{2}} & -\frac{6}{L^{2}} & 0 & 0 & 0 & 0 & 0 \\
0 & \frac{6}{L^{2}} & -\frac{6}{L^{2}} & \frac{4}{L} & -\frac{3}{L} & 0 & 0 & -\frac{6}{L^{2}} & \frac{6}{L^{2}} & \frac{2}{L} & -\frac{3}{L} & 0 & 0 & 0 & 0 & 0 \\
-\frac{1}{L} & 0 & 0 & 0 & 0 & \frac{1}{L} & \frac{1}{L} & 0 & 0 & 0 & 0 & -\frac{1}{L} & 0 & 0 & 0 & 0 \\
0 & -\frac{12}{L^{3}} & \frac{12}{L^{3}} & -\frac{6}{L^{2}} & \frac{6}{L^{2}} & 0 & 0 & \frac{24}{L^{3}} & -\frac{24}{L^{3}} & 0 & 0 & 0 & -\frac{12}{L^{3}} & \frac{12}{L^{3}} & \frac{6}{L^{2}} & -\frac{6}{L^{2}} \\
0 & \frac{6}{L^{2}} & -\frac{6}{L^{2}} & \frac{2}{L} & -\frac{3}{L} & 0 & 0 & 0 & 0 & \frac{8}{L} & -\frac{6}{L} & 0 & -\frac{6}{L^{2}} & \frac{6}{L^{2}} & \frac{2}{L} & -\frac{1}{L} \\
0 & 0 & 0 & 0 & 0 & 0 & -\frac{1}{L} & 0 & 0 & 0 & 0 & \frac{1}{L} & 0 & 0 & 0 & 0 \\
0 & 0 & 0 & 0 & 0 & 0 & 0 & -\frac{12}{L^{3}} & \frac{12}{L^{3}} & -\frac{6}{L^{2}} & \frac{6}{L^{2}} & 0 & \frac{12}{L^{3}} & -\frac{12}{L^{3}} & -\frac{6}{L^{2}} & \frac{6}{L^{2}} \\
0 & 0 & 0 & 0 & 0 & 0 & 0 & \frac{6}{L^{2}} & -\frac{6}{L^{2}} & \frac{2}{L} & -\frac{3}{L} & 0 & -\frac{6}{L^{2}} & \frac{6}{L^{2}} & \frac{4}{L} & -\frac{3}{L}
\end{array}\left|\begin{array}{c}
E A_{1} u_{1} \\
E I v_{1} \\
E I Q v_{1} \\
E I w_{1} \\
E I w_{1} \\
E A_{1} u_{2} \\
E A_{2} u_{2} \\
E I v_{2} \\
E I Q v_{2} \\
E I w_{2} \\
E I Q w_{2} \\
E A_{2} u_{3} \\
E I v_{3} \\
E I Q v_{3} \\
E I w_{3} \\
E I Q w_{3}
\end{array}\right| \begin{array}{c}
H_{1} \\
V_{1} \\
M_{1} \\
H_{2} \\
V_{2} \\
M_{2} \\
H_{3} \\
V_{3} \\
M_{3} \\
y_{3}
\end{array} \mid
\end{aligned}
$$

The next step is to impose the boundary conditions $\left(u_{1}=v_{1}=v_{3}=0\right)$ and include the measured deflections and rotations $\left(w_{1}, u_{2}, v_{2}\right.$ and $\left.w_{2}\right)$, removing them from vector $\delta_{2}^{*}$ and introducing them into the modified stiffness matrix $\left[K_{3}{ }^{*}\right]$ to obtain Equation (16). In this way, the columns associated with measured variables are multiplied by their corresponding values and the associated factors are removed from the vector of variables $\left\{\delta_{3}^{*}\right\}$. The modified system after incorporating the boundary conditions and the measured variables is as follows:

$\left[K s_{3}^{*}\right] \cdot\left\{\delta_{3}^{*}\right\}=\{f\}$

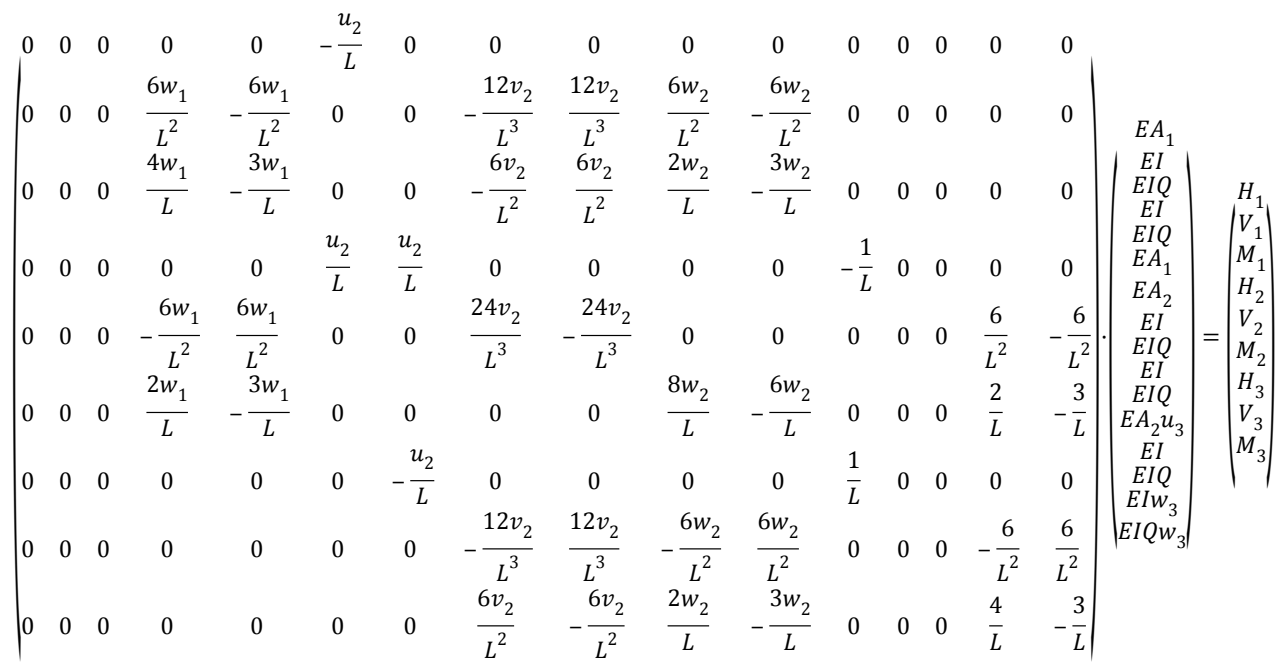


The next step showed in Equation (17) consists of clustering together the repeated coupled variables in the vector of unknowns $\left\{\delta_{4}^{*}\right\}$ by joining together the corresponding columns of $\left[K s_{3}{ }^{*}\right]$. In this example, this is the case of the columns related to $E A_{1}, E I$ and $E I Q$. If after this process null columns are obtained, these columns will be removed together with the corresponding variables from $\left\{\delta_{4}^{*}\right\}$ to obtain a simpler matrix.

$$
\left[K s_{4}^{*}\right] \cdot\left\{\delta_{4}^{*}\right\}=\{f\}
$$

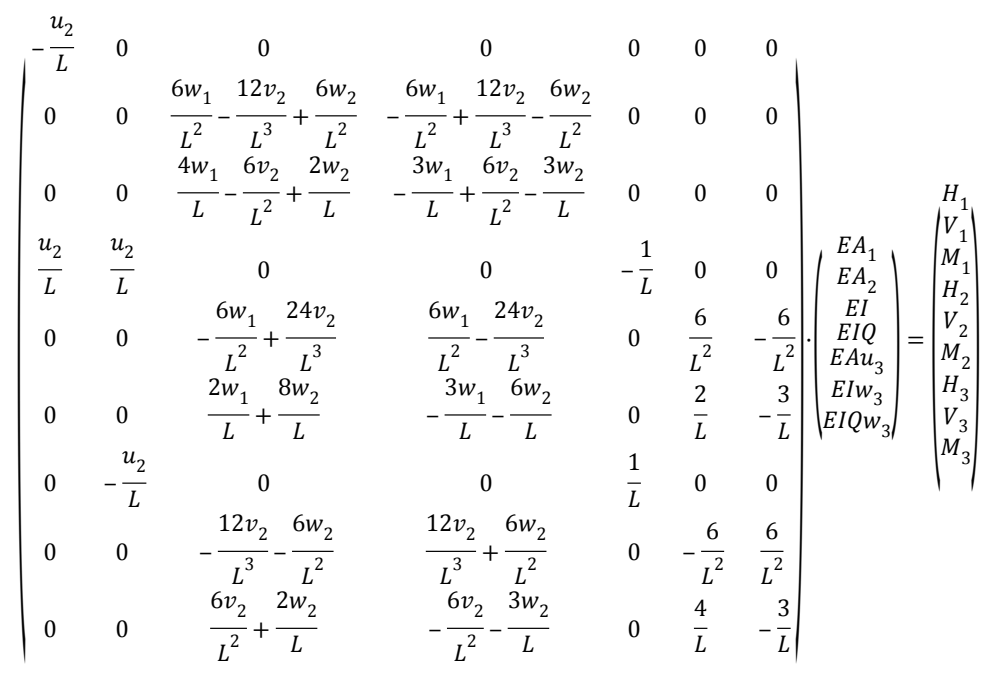

According to (4) the system (17) can be rearranged as follows:

$[B] \cdot\left\{z_{1}\right\}=\{D\}$

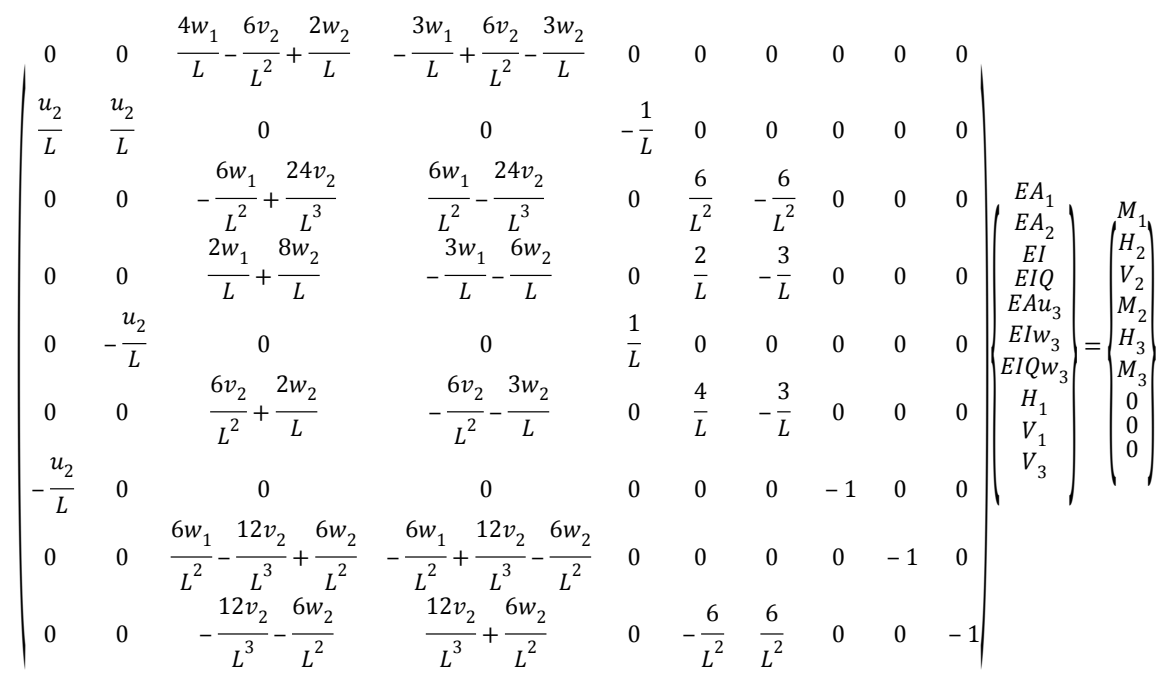

Matrix $[B]$ is formed by the partitioned matrices $k_{00}^{*}$ and $k_{10}^{*}$ and the null and the identity matrices. The vector $\left\{z_{1}\right\}$ includes the reactions due to the boundary conditions $\left(H_{1}, V_{1}\right.$ and $\left.V_{3}\right)$, the axial stiffnesses $\left(E A_{1}\right.$ and $\left.E A_{2}\right)$, the flexural stiffness $(E I)$, the observability shear stiffness $(E I Q)$ and nonlinear products of axial, flexural and observability shear stiffnesses coupled with deflections and rotations $\left(E A_{2} u_{3}, E I w_{3}\right.$ and $\left.E I Q w_{3}\right)$.

Afterwards, the null space $[V]$ of $[B]$ has to be obtained. Then, the system can be expressed in terms of a particular solution \{ $\left.z_{p 1}\right\}$ added to the null space $[V]$ multiplied by a vector of coefficients $\left\{p_{1}\right\}$. The analysis of the null space leads to determine the observable variables (variables with a unique solution) by identifying the null rows of the null space. Hence, the general solution of $\left\{z_{1}\right\}$ for this first recursive step is as follows: 
$\left.\left\{z_{1}\right\}=\left\{z_{p 1}\right\}+[V] \cdot\left\{p_{1}\right\}=\left(\begin{array}{c}\boldsymbol{E} \boldsymbol{A}_{\mathbf{1}} \\ E A_{2} \\ \boldsymbol{E I} \\ \boldsymbol{E I Q} \\ E A_{2} u_{3} \\ \boldsymbol{E I w _ { 3 }} \\ \boldsymbol{E I Q \boldsymbol { w } _ { 3 }} \\ \boldsymbol{H}_{\mathbf{1}} \\ \boldsymbol{V}_{\mathbf{1}} \\ \boldsymbol{V}_{\mathbf{3}}\end{array}\right)=\left\{z_{p 1}\right\}+\mid \begin{array}{c}0 \\ 1 / u_{2} \\ 0 \\ 0 \\ 1 \\ 0 \\ 0 \\ 0 \\ 0 \\ 0\end{array}\right) \cdot\left\{p_{1}\right\}$

The general solution is the set of all possible solutions to the system. The solution for all the unknown parameters of the structure includes a particular solution and the set of all solutions of the associated homogeneous system of equations. Therefore, the determination of the null rows of the null space leads to determine the observable parameters (highlighted in bold in Equation (19)).

The last step is to calculate the numeric value of the observed parameters. This solution can be symbolically obtained from the parametric equations of $[B]$. Once identified the observed parameters, their value can be numerically calculated. The parametric equations are presented as follows:

$$
\begin{aligned}
& \widehat{E A_{1}}=\frac{H_{2} L}{u_{2}} \\
& \hat{E I}=\frac{L^{2} V_{2}}{4\left(w_{1}-w_{2}\right)} \\
& \widehat{E I Q}=\frac{L\left(2 L^{2} V_{2} w_{1}-3 L V_{2} v_{2}+L^{2} V_{2} w_{2}\right)}{6\left(w_{1}-w_{2}\right)\left(L w_{1}-2 v_{2}+L w_{2}\right)} \\
& \hat{Q}=\frac{4 L w_{1}-6 v_{2}+2 L w_{2}}{3 L w_{1}-6 v_{2}+3 L w_{2}}
\end{aligned}
$$

For the first time in the literature, a parametric equation of $\widehat{E I Q}$ depending on the measured data (Equation (22)) is obtained. The parametric equation of $\hat{E} I$ is calculated by Equation (22), can be used to obtain the shear parameter $\hat{Q}$ as presented in Equation (23). This equation shows that $\hat{Q}$ can only be determined when the value of the corresponding flexural stiffness is known. Finally, once the values of the observable variables are calculated, the observability shear stiffness can be expressed in terms of $\left(E A_{v}\right)$ using Equation (11). Finally, as new information is obtained from observable parameters in the current analysis (recursive step), this information can be reintroduced in the system. This recursive process would continue until no additional variables are observed between two subsequent steps.

To illustrate the required measurement set to obtain the full observability of a structure, an example with a more complex structure, a simple supported beam with more unknowns is presented in the following section.

\subsection{Example 2 with the new formulation}

To evaluate the application of the observability analysis in structures with a higher number of unknowns and to define the characteristics of the required measurement set to achieve full observability of a structure, the simply supported beam depicted in Figure 2.A is analyzed. The targeted unknowns are: $\left(E I_{1}, E I_{2}, E I_{3}, E A v_{1}, E A v_{2}\right.$ and $\left.E A v_{3}\right)$. The mechanical and geometrical properties of the analyzed example corresponds with the beam presented in Section 2.2, The deformed shape including shear deformation is presented in Figure 2.B. Different sets including vertical deflections, $v s$, and rotations, ws, obtained by this simulation are introduced as input data.

All possible sets made out of 3 to 12 measurements were analyzed. Results are presented in Figure 5. This figure includes how many times a unique solution (observability) is obtained when the same number of measurements is used.

Figure 5 shows, from all the possible measurement sets, the percentage of sets able to obtain the solution for all the unknown flexural stiffness $(E I)$ and shear stiffness $(E A v)$. This illustrates that no set with 3 measurements provides any result. However, when the number of measurements per set is increased to 4, a small number of combinations (4.24\%) is able to obtain all the EI. All of these combinations are always composed by 4 rotations, $w$.

If the set is composed by more measurements, the percentage of sets providing full observability increases. For example: in the case of the sets with 6 measurements, the total number of possible combinations is 924 and the number of combinations enabling the observability of all the unknown parameters is only 168 . Therefore, only the $18.18 \%$ of the combinations with 6 measurements provides the values all the unknown parameters. All the adequate combinations for this set are composed by 2 vertical displacements, $v$, and 4 rotations, $w$. For the sets of 7 measurements, the total number of possible combinations is 792 and the number of observable combinations is 305 . So, the percentage of useful combinations increases to $38.51 \%$. Moreover, even in the case of the sets of 7 
measurements, the minimum number of rotations, $w$, is always 4 . Thus, it can be said that, for this problem, 4 are the least possible number of rotations needed to determine all the unknown parameters.

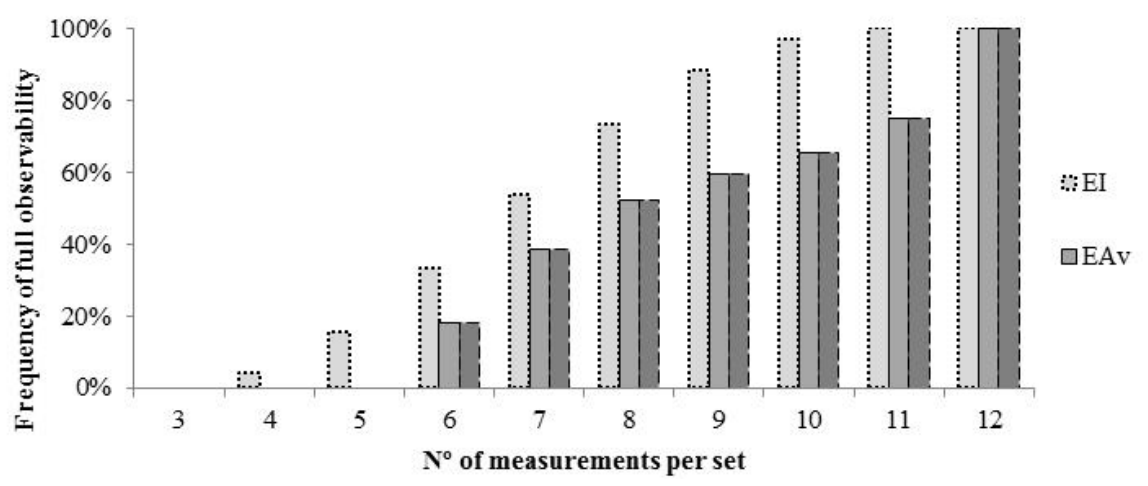

Figure 5: Frequency of observability for Ei, EAv and EI+EAv for all possible combinations of different number of measurements.

Figure 5 also shows that no shear $(E A v)$ parameter can be obtained unless the corresponding (EI) is first observed. Furthermore, to fully observe the 6 structural unknowns, at least six measurements have to be introduced as input. Thus, any set with less than 6 measurements is inadequate to find all the unknown variables.

In order to show the results obtained, some representative sets have been selected and presented in Table 1. This table also shows the unknown variables obtained by using the observability algorithm.

Table 1: Observable variables for different measurement sets

\begin{tabular}{cc|cccccc}
\hline & & \multicolumn{7}{|c}{ Parameters observed } \\
& Met & $\boldsymbol{E} \boldsymbol{I}_{\mathbf{1}}$ & $\boldsymbol{E I}_{\mathbf{2}}$ & $\boldsymbol{E} \boldsymbol{I}_{\mathbf{3}}$ & $\boldsymbol{E} \boldsymbol{A}_{\boldsymbol{v}_{\mathbf{1}}}$ & $\boldsymbol{E}_{\boldsymbol{v}_{\mathbf{2}}}$ & $\boldsymbol{E}_{\boldsymbol{v}_{\mathbf{3}}}$ \\
\hline 1 & $v_{2}, w_{1} \& w_{2}$ & $\checkmark$ & $\mathbf{x}$ & $\mathbf{x}$ & $\checkmark$ & $\mathbf{x}$ & $\mathbf{x}$ \\
2 & $v_{2}, v_{3}, v_{4}, v_{5}, v_{6}, w_{1} \& w_{2}$ & $\checkmark$ & $\mathbf{x}$ & $\mathbf{x}$ & $\checkmark$ & $\mathbf{x}$ & $\mathbf{x}$ \\
3 & $v_{2}, v_{3}, v_{4}, v_{5} \& v_{6}$ & $\mathbf{x}$ & $\mathbf{x}$ & $\mathbf{x}$ & $\mathbf{x}$ & $\mathbf{x}$ & $\mathbf{x}$ \\
4 & $v_{2}, v_{3}, v_{4}, v_{5}, v_{6} \& w_{1}$ & $\mathbf{x}$ & $\mathbf{x}$ & $\mathbf{x}$ & $\mathbf{x}$ & $\mathbf{x}$ & $\mathbf{x}$ \\
5 & $w_{1}, w_{2}, w_{3}, w_{4}, w_{5} \& w_{6}$ & $\checkmark$ & $\checkmark$ & $\checkmark$ & $\mathbf{x}$ & $\mathbf{x}$ & $\mathbf{x}$ \\
6 & $w_{1}, w_{3}, w_{5}, w_{7}, v_{4} \& v_{6}$ & $\checkmark$ & $\checkmark$ & $\checkmark$ & $\checkmark$ & $\checkmark$ & $\checkmark$
\end{tabular}

Sets $1\left(v_{2}, w_{1} \& w_{2}\right)$ and $2\left(v_{2}, v_{3}, v_{4}, v_{5}, v_{6}, w_{1} \& w_{2}\right)$ show that additional measurements $\left(v_{4}, v_{5}, v_{6}\right)$ do not mean necessarily obtaining more variables. The importance of selecting the correct measurement set is highlighted. Set 3 and 4 show that the measurement of all displacements gives little information if it is not combined with enough rotations. However, measurement set 5 , which only includes rotations, gives an accurate result for all the flexural stiffnesses but does not provide the information to observe any of the $(E A v)$. Finally, measurement set 6 is able to determine all the observable parameters. This measurement set is composed by 4 rotations $\left(w_{1}, w_{3}, w_{5}\right.$ and $\left.w_{7}\right)$ and 2 vertical displacements

Hence, the new formulation is able to find the correct solution of the observability variables when the correct set of measurements is used. The set of measurements to find all the flexural stiffnesses and the observability shear stiffnesses of the beam must include information about rotations and vertical displacements. This information is required to uncouple all the unknown parameters properly. Furthermore, only with rotations the algorithm is unable to determine the observability shear stiffnesses. Thus, it is essential to choose the right combination of rotations and deflections to determine all the unknown parameters.

Accurate estimations of all the observed parameters are obtained for all the analyzed sets when the shear deformation is included into the formulation and numerical free-error measurements are considered.

\section{APPLICATION TO THIN WEB BRIDGES}

Accurate models to control construction of cantilever bridges are of uttermost importance. Deflection should be both controlled and forecast in order to give an adequate geometry to the segments. Hence, to calculate precamber, an accurate model upon which an analysis has to be performed is needed. As an example on how to use this technique to help model updating, a simplified model of the Yunbao Bridge over the Yellow Riverin China (see Fig. 6.A) will be studied. The span of the structure is $90 \mathrm{~m}$ long. The model represents a simplification of an intermediate construction stage. This model includes two symmetric spans in cantilever supported by an intermediate pile. The analyzed construction stage considers balanced cantilevers $13.5 \mathrm{~m}$ long, composed by three deck segments of $4.5 \mathrm{~m}$. A detail of the steel web of the composite section is presented in Fig. 6.B. The structural response at this stage is simulated numerically without considering actual site data. The creep and shrinkage effects in concrete were neglected. 

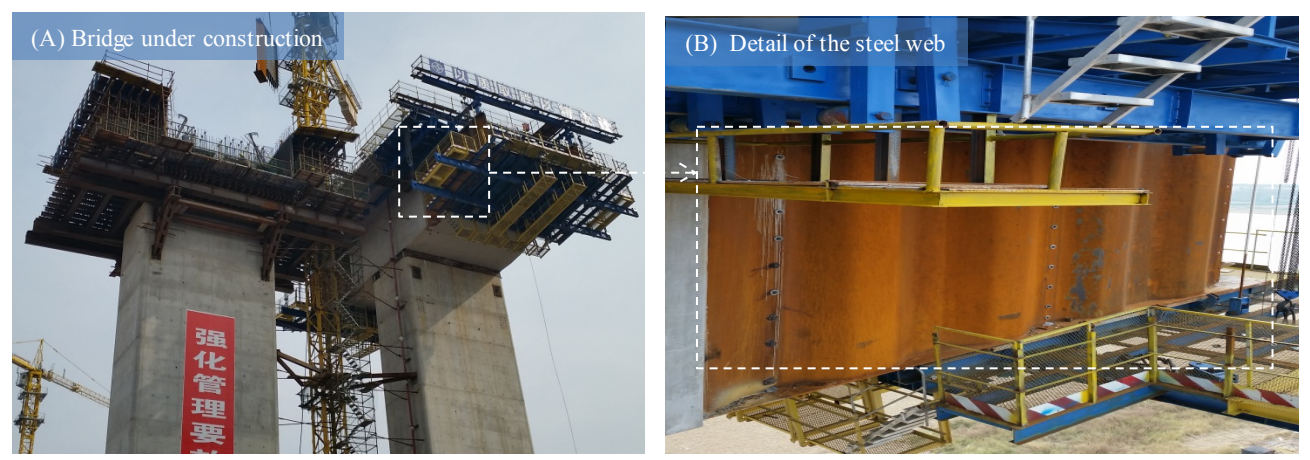

Figure 6: Composite bridge on site in China. (A) Cantilever erection and (B) Detail of the steel web.

This structure is simulated by the mean of the simplified FEM presented in Fig. 7.A. This FEM includes 8 nodes and 7 beam elements. The cross section of the composite deck is presented in Fig. 7.B and the mechanical and material properties are listed in Table 2. Whereas in the real bridge webs are corrugated, in the analyzed example they are considered $25 \mathrm{~mm}$ thick steel sheets. The connection between concrete and steel is assumed as rigid, and the relative slip between both materials is neglected. In order to make the calculations of the theoretical properties of the composite cross section, full interaction is considered (no slip at the interphase between steel and concrete). In order to evaluate axial and bending stiffness any formulation of strength of materials of composite sections can be used. However, as the axial stiffness of the corrugated web is smaller than the one of the straight web due to the accordion effect, this has to be taken into account. In order to do so, simple finite element models or Castigliano theorem can be used to calculate the reduction of axial stiffness that crippled webs imply $[55,56]$. According to these calculations axial and bending mechanical properties of the steel part of the composite section of this particular bridge should be multiplied by 0.001136 . Hence, the steel section contributes very little to the axial and bending stiffness of the composite cross section. It is assumed that shear is transferred by the steel webs. Crippled web also reduces shear stiffness compared with straight webs as the actual length of the web is bigger than the one of the segment. In order to take this into account in a model made out of straight elements, shear stiffness of the crippled web has to be reduced so as to take into account the real length of the web. Shear stiffness $K_{S}$ can be calculated according to the following equation

$$
K_{s}=\frac{V}{\gamma}=G \cdot A_{q} \cdot \frac{L_{s}}{L_{w}}
$$

Where $\mathrm{V}$ is the shear force, $\gamma$ is the shear distortion, $L_{s}$ is the longitudinal length of the segment or the length of the longitudinal axis of the corrugated web and $L_{w}$ is the actual length of the web, considering the cripples. Shear stiffness of the crippled web is reduced by 0.879 . The properties in Table 2 were defined by the method of the transformed section [57].

Studies (such as [58]) show that the classical beam theory fails to reproduce the shear force flow near the joints and supports. This phenomenon is neglected in this study. Nevertheless, for a more accurate simulation, K-truss mechanism [59, 60] can be introduced in the model.

(A) Finite Element Model

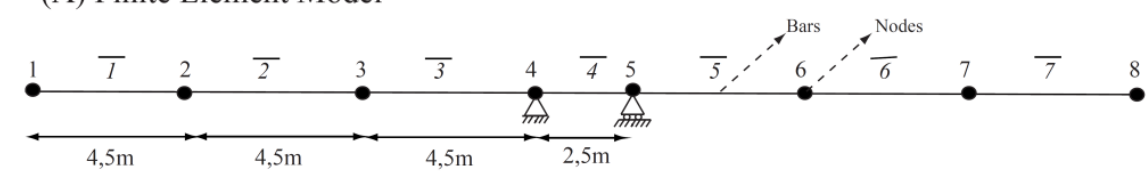

(B) Cross section

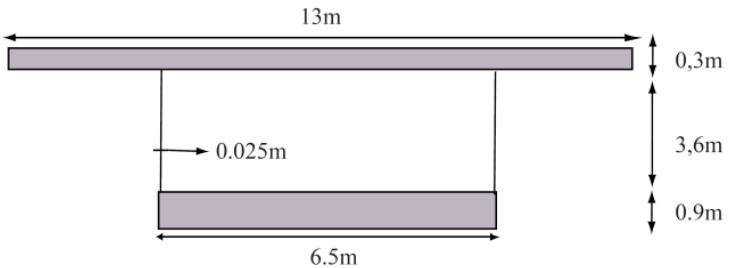

Figure 7: Bridge in China (A) Finite Element Model and geometry. (B) Cross Section.

Table 2: Properties of the Finite Element Model of the Bridge

\begin{tabular}{l|l}
\hline Area, $\mathrm{m}^{2}$ & 12.59 \\
Shear Area, $\mathrm{m}^{2}$ & 9.83 \\
Inertia, $\mathrm{m}^{4}$ & 35.62 \\
Steel Young's Modulus, GPa & 210 \\
Concrete Young's Modulus, GPa & 35 \\
Poissons' ratio, $v$ & 0,3
\end{tabular}


The flexural and shear stiffnesses of all 7 beam elements (that is to say $E I_{1}$ to $E I_{7}$ and $E A v_{l}$ to $E A v_{7}$ ) are assumed as unknown.

\subsection{Static load test}

In order to identify the 14 unknown stiffnesses the measurement of 14 deflections and/or rotations in a static load case that excites the flexural mechanism is required. Observability will take into account the variation of deflections and rotations due to the movement of the form traveler. The load case used is derived from Fig. 8. There, it is assumed that the weight $\mathrm{Q}$ of the formwork traveler (weight of formwork included) is around $60 \%$ of the weight of the segment $(1041 \mathrm{kN})$. The effect of each form traveler in the deck is assumed to be a pair of vertical forces of values 0.25Q and 1.25Q (226 kN, upwards and 1267 kN, downwards). Load case used for the SSI is calculated by deducing the effects of the stage i (Fig. 8.A), from stage i+1 (Fig. 8.B), in which the formwork is moved forward to the construction of the next segment. The resulting load case in Stages $\mathrm{i}$ and $\mathrm{i}+1$ are presented in Figures 8 .C and 8.D, respectively. The resultant load case introduced in the simulation is shown in Fig. 8.E. In this paper, measurement errors are neglected. The effect of these unavoidable errors will be addressed by the authors in future works

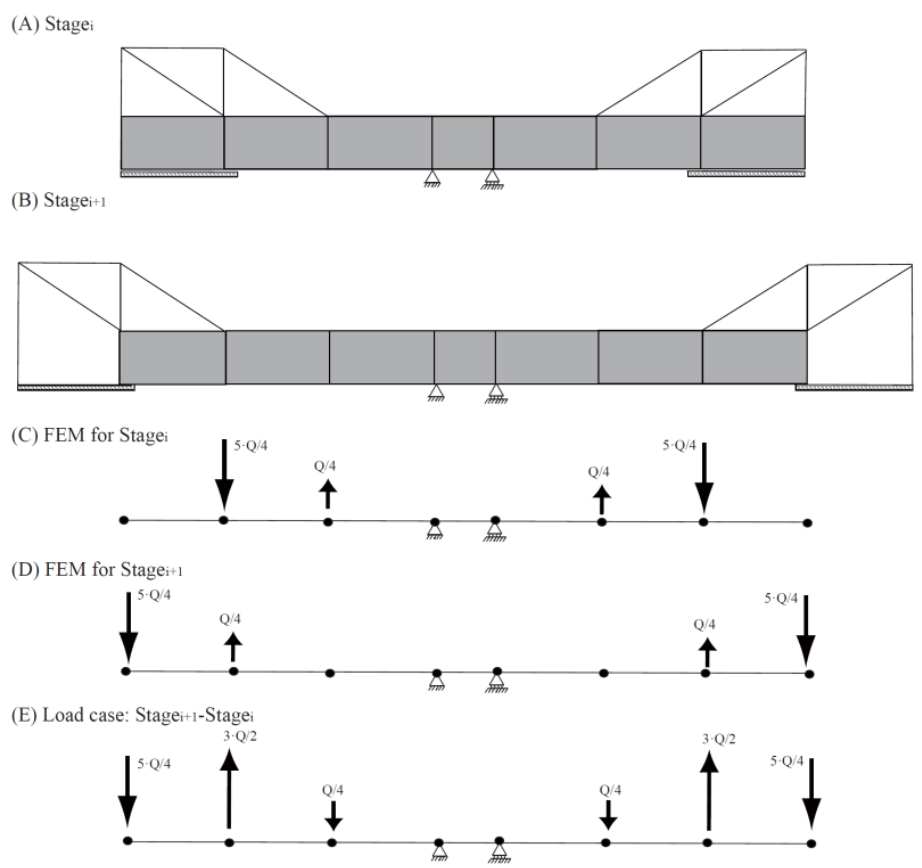

Figure 8: Definition of the load case: $(A, C)$ Stage i. (B, D) Stage i+1, (E) Load case used for the inverse analysis.

The vertical deflections in the nodes of the structure are calculated with the software Midas/Civil [45] both without including and including the shear deformation of the deck. Fig. 9 includes a comparison of both deflections.

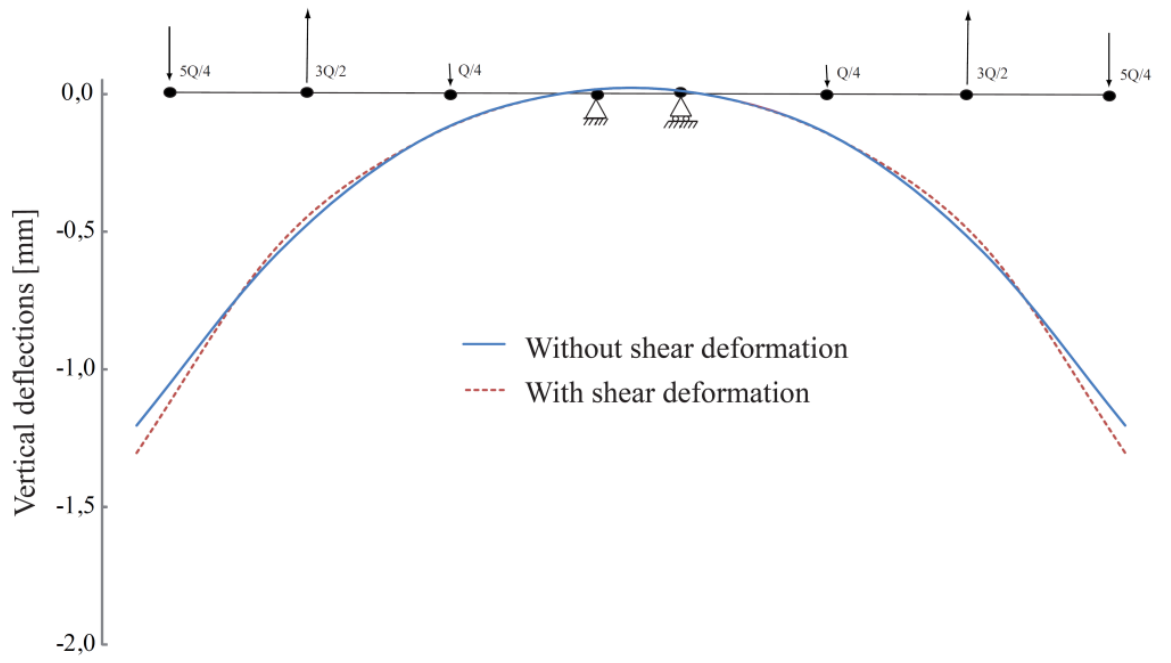

Figure 9: Variation of vertical deflections at an intermediate construction stage with and without shear deformation

This figure shows that the maximum deflection variations are located at the tips of the cantilevers. Shear deformation accounts for $0,1 \mathrm{~mm}$ of the vertical displacement, which is not negligible as it represents the $7,6 \%$ of the total deformation. To get accurate measurements of the vertical deflections on site, different measuring devices can be used. An example of these devices, valid for static and dynamic identification [61] is the measurement system proposed in Ribeiro et al. [62]. In this procedure, a precision of $0.067 \mathrm{~mm}$ can be achieved on site conditions. 


\subsection{SSI by observability techniques}

In this section the measured response of the structure is used to estimate the unknown stiffnesses. A flexural observability tree [42] has been used to select an adequate measurement set. When shear stiffness is neglected the measurement set consists on all the deflections $\left(\mathrm{v}_{1}, \mathrm{v}_{2}, \mathrm{v}_{3}, \mathrm{v}_{6}, \mathrm{v}_{7}, \mathrm{v}_{8}\right)$ and one rotation $\left(\mathrm{w}_{4}\right)$. On the other hand, when shear stiffnesses is considered in the formulation the rest of rotations are also required. The rotations can be measured with inclinometers with high resolution. For example, the device 210/220 from the German company Leica has a resolution of 1e-6 rad [63]. This type of inclinometer has been applied in the construction control of the vertical alignment of some skyscrapers, such as World Trade Center [64].

Considering the shear stiffness, parametric equations can be obtained for the different elements. The equations of the stiffnesses at some beam elements, $\widehat{E I}$ and $\widehat{E I Q}$, are presented.

$$
\begin{aligned}
& \hat{E} I_{1}=\frac{-81 L^{2} V_{1}}{8\left(w_{1}-w_{2}\right)} \\
& \hat{E} I_{2}=\frac{-9 L\left(-27 L V_{1}+9 L V_{2}\right)}{8 \cdot\left(w_{2}-w_{3}\right)} \\
& \hat{E} I_{3}=\frac{-9 L\left(-45 L V_{1}-27 L V_{2}+9 L V_{3}\right)}{8 \cdot\left(w_{3}-w_{4}\right)} \\
& \hat{E} I_{4}=\frac{-5 L\left(27 L V_{1}+18 L V_{2}+9 L V_{3}\right)}{8 \cdot\left(w_{4}-w_{5}\right)} \\
& \hat{E I} Q_{1}=\frac{9 L\left(-18 L V_{1} v_{1}+18 L V_{1} v_{2}-54 L^{2} V_{1} w_{1}-27 L^{2} V_{1} w_{2}\right)}{4\left(w_{1}-w_{2}\right) \cdot\left(4 v_{1}-4 v_{2}+9 L w_{1}+9 L w_{2}\right)}
\end{aligned}
$$

These equations can be used to determine how sensitive the different estimates are to errors.

Figure 10 presents a comparison of the differences between the estimated flexural stiffnesses $(\hat{E I})$ and the actual values $(\mathrm{EI})$ in the seven elements of the beams with two observability procedures (with or without the shear effects into the stiffness matrix).

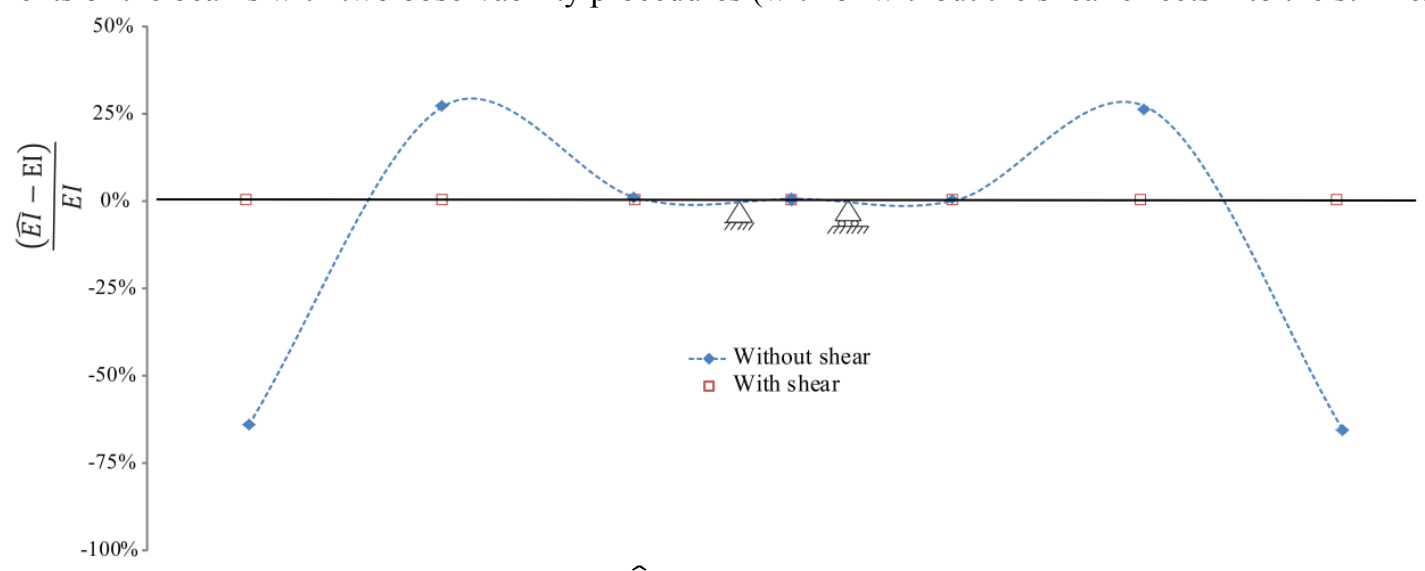

Figure 10: Percentage deviation of $\widehat{E I}$ without and with shear deformation in the SSI.

This figure shows that deviations when the shear formulation is included into the stiffness matrix and error-free measurements are considered are negligible. The reduced deviations obtained in this case can be explained by the fact numerical and non-actual site measurements are considered. It also illustrates that shear deformation cannot be neglected for the structural system identification of this structure. In fact, significant errors (e.g. $-65,5 \%$ in segments 1 and 7 or $+26,7 \%$ in segments 2 and 6 ) appear when this phenomenon is not considered.

\section{CONCLUSIONS}

Most Structural System Identification (SSI) methods neglect shear deformation as this phenomenon is usually much less significant than the flexural one. Despite of the important role that this deformation might play especially in members with low span-to-depth ratio, no detailed study addressing the particular effects of this deformation in static SSI tests can be found in the literature. To fill this gap, this paper presents the first study focused on the shear deformation effects in a structural system identification method: the observability technique. This is a parametric method based on static measurements.

An illustrative example shows how important the role of the shear deformation might be. In fact, this example illustrates that 
the difference between Timoshenko's theory and Euler-Bernoulli theory is remarkable for members with moderate span-to-depth ratio. To solve this modeling error, the formulation of the observability method is updated to include shear deformation. A detailed step by step example is presented to illustrate the algorithm. This new procedure enables, for the first time in the literature, the definition of parametric equations of the observability shear stiffness depending on the measured data for simple structures. The study of this equation might provide useful insight of the sensitivity to the estimation for each measurement. The proposed technique is also applied into a simply supported beam with six unknown parameters. This example proves the applicability of the observability techniques in actual structures including shear deformation, providing that enough information is obtained from a static test. Rotations and deflections should be measured in order to get full observability (bending and shear stiffness). If only the bending stiffness is targeted, rotations can provide enough information to get so.

In order to show the applicability of the method on an actual thin web structure, an intermediate construction stage of a cantilever composite bridge in China is analyzed. The results of this structure illustrate how an adequate formulation can be used to reduce adequately the modeling errors without the need of optimization processes. This updated formulation enables also the calculation of parametric equations of the estimates that gives us information about their sensitivity to measurement errors.

\section{ACKNOWLEDGEMENT}

The authors are indebted to the Spanish Ministry of Economy and Competitiveness for the funding provided through the research project BIA2013-47290-R founded with FEDER funds and directed by Jose Turmo.

\section{REFERENCES}

1. Sanayei, M., Onipede, O., and Babu, S.R. Selection of noisy measurement locations for error reduction in static parameter identification. AIAA Journal, 30 (1992) 2299-2309.

2. Sanayei, M. and Saletnik, M.J. Parameter estimation of structures from static strain measurements I: Formulation. Journal of Structural Engineering, 122 (1996) 555-562.

3. Yan, A., Golinval, J. Structural damage localization by combining flexibility and stiffness methods. Engineering Structures 27 (2005) $1752-1761$.

4. Liao, J., Tang, G., Meng, L., Liu, H., and Zhang, Y. Finite element model updating based on field quasi-static generalized influence line and its bridge engineering application. Procedia Engineering, 31 (2012) 348-353.

5. Chang, K.C., Kim, C.W. and Kawatani, M. Feasibility investigation for a bridge damage identification method through moving vehicle laboratory experiment. Structure and Infrastructure Engineering, 10 (2014) 328-345.

6. Chao, S.H. and Loh, C.H. Application of singular spectrum analysis to structural monitoring and damage diagnosis of bridges. Structure and Infrastructure Engineering, 10 (2014) 708-727.

7. Banan, M.R. \& Hjelmstad, K.D. Identification of structural systems from measured responses. (Technical report, Report No. SRS 579, UILUENG 93-2002). Urbana, IL, 1993, University of Illinois at Urbana-Champaign.

8. Jung, S., Ok, S.-Y. and Song, J. Robust structural damage deification based on multi-objective optimization. International Journal for Numerical Methods in Engineering, 81 (2010) 786-804.

9. Ren, W.X., Fang, S E. and Deng, M.Y. Response surface-based finite-element-model updating using structural static responses. Journal of Engineering Mechanics, 137 (2011) 248-57.

10. Sanayei, M., and Onipede, O. Damage assessment of structures using static test data. AIAA Journal, 29 (1991) $1174-1179$.

11. Banan, Mo.R., Banan, Ma.R., and Hjelmstad, K.D. Parameter estimation of structures from static response. I. Computational aspects. Journal of Structural Engineering, 120 (1994a) 3243-3258.

12. Banan, Mo.R., Banan, Ma.R., and Hjelmstad. K.D. Parameter estimation of structures from static response. II: Numerical simulation studies. Journal of Structural Engineering, 120 (1994b) 3259-3283.

13. Sanayei, M., Phelps, J.E., Sipple, J.D., Bell, Erin S., and Brenner, Brian R. Instrumentation, nondestructive testing, and finite-element model updating for bridge evaluation using strain measurements. Journal of Bridge Engineering, 17 (2012) 130-138.

14. Abdo, M.A. Parametric study of using only static response in structural damage detection. Engineering Structures, 34 (2012) $124-131$.

15. EN 1992-1-1:Eurocode 2: Design of concrete structures - Part 1-1: General rules and rules for buildings. CEN, Brussels, 2002, Belgium.

16. ACI committee 318 Building code requirements for structural concrete and commentary. American Concrete Institute, Detroit, 2000, USA.

17. Noroozinejad Farsangi, E., Melatdoust, H. and Bin Adnan, A.A mathematical formulation to estimate the fundamental period of highrise buildings including flexural-shear behavior and structural interaction. Journal of Solid Mechanics, 6 (2014) 122-134.

18. Lin, K.C., Hung, H.H. \& Sung, Y.C. Seismic Performance of High Strength Reinforced Concrete Buildings Evaluated by Nonlinear Pushover and Dynamic Analyses. International Journal of Structural Stability and Dynamics. 16 (2016) 1-27.

19. Li, G., Hao, K., Lu, Y. and Chen, S.A flexibility approach for damage identification of cantilever-type structures with shear deformation. Computers and Structures 73 (1999) 565-572.

20. Ebrahimian, M. and Todorovska, M.I. Structural System Identification of Buildings by a Wave Method Based on a Nonuniform Timoshenko Beam Model. J. Eng. Mech., 141(2015) 04015022 1-11.

21. Kang, J.S., Park, S., Shin, S. and Lee, H.S. Structural system identification in time domain using measured acceleration. Journal of Sound and Vibration, 288 (2005) 215-234.

22. Lei, Y., He, M., Liu, C. and Lin, S. Identification of Tall Shear Buildings under Unknown Seismic Excitation with Limited Output Measurements. Advances in Structural Engineering, 16 (2013) 1839-1850.

23. Sahraei A. and Mohareb M. Upper and lower bound solutions for lateral-torsional buckling of doubly symmetric members, Thin-walled structures, 102 (2016) 180-196.

24. Bhat S.U. de Oliveira J.G Formulation for the Shear Coefficient of Thin-Walled Prismatic Beams, Journal of Ship Research, 29 (1985) $51-58$.

25. Chen, H. Blandford, G.E. A finite element formulation for thin-walled beams, Int J Numer Methods Eng, 20 (1989) $2239-2255$.

26. Back, S.Y. Will, K.M. A shear-flexible element with warping for thin-walled open beams, Int J Numer Methods Eng, 43 (1989) $1173-$ 1191. 
27. Shakourzadeh, H. Guo, Y.C. Batoz J.L. A torsional bending element for thin-walled beams with open and closed cross sections, Comput Struct, 55 (1995) 1045-1054.

28. Erkmen, R.E., Mohareb, M. Torsion analysis of thin-walled beams including shear deformation effects, Thin-Walled Structures, 44 (2006) 1096-1108.

29. Van Phan, P. and Mohareb, M. A shear deformable theory for the analysis of steel beams reinforced with GFRP plates. Thin-walled structures, 85 (2014) 165-182.

30. Erkmen R.E. Shear deformable hybrid finite-element formulation for buckling analysis of thin-walled members, Finite Elem.Anal. Des., $82(2014) 32-45$

31. Poul, M. Khazaei; N.F.; Zhao, X.L. Experimental testing on CFRP strengthened thin steel plates under shear loading, Thin-walled structures, 109 (2016) 217 -226.

32. Chen, D. Kitipornchai, S.; Yang, J. Nonlinear free vibration of shear deformable sandwich beam with a functionally graded porous core, Thin-walled structures, 107 (2016) 39-48.

33. Hossain, K.M.A. Rafiei, S. Lachemi, M. et al. Finite element modeling of impact shear resistance of double skin composite wall, Thinwalled structures, 107(2016) 101-118.

34. Tong, G. Feng, Y., Tao, W. et al. Elastic stability of plate simply supported on four sides subjected to combined bending shear and patch loading, Thin-walled structures, 107 (2016) 377-396.

35. Rasool, M. Singha, M.K.A. finite element study on the nonlinear behavior of rectangular shear panels, Thin-walled structures, 104 (2016) 248-258.

36. Kim, N.I. Choi, D.-H. Super convergent shear deformable finite elements for stability analysis of composite beams, Compos. Part B: Eng., 44 (2013) 100-111.

37. Henriques, D. Goncalves, R., Comotim, D. GBT-based finite element to assess the buckling behaviour of steel-concrete composite beams, Thin-walled structures, 107 (2016) 207-220.

38. Sabouri-Ghomi, S.; Jahani, Y.; Bhowmick, A.K. Partial interaction theory to analyze composite (steel-concrete) shear wall systems under pure out-of-plane loadings, Thin-walled structures, 104 (2016) 211-224.

39. Zhou, M., Liu, Z., Zhang, J., An, L. Deformation analysis of a non-prismatic beam with corrugated steel webs in the elastic stage, ThinWalled Structures, 109 (2016) 260-270.

40. Leblouda, M., Junaid, M.T., Barakat, S., Maalej, M. Shear buckling and stress distribution in trapezoidal web corrugated steel beams; Thin-walled structures, 113 (2017) 13-26.

41. Lozano-Galant, J.A., Nogal, M., Paya-Zaforteza, I., and Turmo, J. Structural system identification of cable-stayed bridges with observability techniques. Structure and Infrastructure Engineering, 10 (2014) 1331-1344.

42. Lozano-Galant, J.A., Nogal, M., Turmo, J., and Castillo, E. Selection of measurement sets in static structural identification of bridges using observability trees. Computers and Concrete, 15 (2015) 771-794.

43. Lei, J., Lozano-Galant, J.A., Nogal, M., Xu, D., Turmo, J.. Analysis of measurement and simulation errors in structural system identification by observability techniques. Structural Control and Health Monitoring, (2016) doi: 10.1002/stc.1923.

44. Lei J, Lozano-Galant, J.A., Nogal, M., Xu, D., Turmo, J. Constrained observability method in structural system identification, Structural Control and Health Monitoring, Structural Control and Health Monitoring 2017: e2040. https://doi.org/10.1002/stc.2040

45. Midas Civil [Computer software] Midas Information Technology Co.2015, Ltd. http://en.midasuser.com/product/civil_overview.asp.

46. Castillo, E., Lozano-Galant, J.A., Nogal, M. and Turmo, J. New tool to help decision making in civil engineering. Journal of Civil Engineering and Management, 21 (2016a) 689-697.

47. Castillo, E., Nogal, M., Lozano-Galant, J.A. and Turmo, J. Solving Some Special Cases of Monomial Ratio Equations Appearing Frequently in Physical and Engineering Problems. Mathematical Problems in Engineering, (2016b) http://dx.doi.org/10.1155/2016/9764913

48. Castillo, E., Jubete, F., Pruneda, R.E. and Solares, C. Obtaining simultaneous solutions of linear subsystems of equations and inequalities Linear Algebra and Its Applications, 346 (2002) 131-54.

49. Matlab [Computer software]. The MathWorks I. Matlab Primer. 2015a ed. 2015, https://www.mathworks.com/help/pdf_doc/matlab/getstart.pdf

50. Nogal, M., Lozano-Galant, J.A., Turmo, J. and Castillo, E. Numerical damage identification of structures by observability techniques based on static loading tests, Structure and Infrastructure Engineering, 12 (2015) 1216-1227.

51. Lozano-Galant, J.A., Nogal, M., Castillo, E. and Turmo, J. Application of Observability Techniques to Structural System Identification. Computer-Aided Civil and Infrastructure Engineering 28 (2013) 434-450.

52. Lei, J., Xu, D., Turmo, J. Static structural system identification for beam-like structures using compatibility conditions Structural Control and Health Monitoring 2017 :e2062. https://doi.org/10.1002/stc.2062

53. Renton, J.D. Generalized beam theory applied to shear stiffness. Int. J. Solids Struct., 27 (1991) 1955-1967

54. Przemieniecki, J.S.Theory of Matrix Structural Analysis. Library of Congress Catalog Card Number 67(1968), 19151

55. Xu, D., Zhao, L., Xu, Z., Du, S., Wang, X., Jin, W. Construction of the Yunbao bridge over the Yellow river Proceedings of the East Asia-Pacific Conference on Structural Engineering and Construction (EASEC 15) Xi'an, China, October 11-13, 2017.

56. Xu, D., Ni, Y., Zhao, Y. (2015). Analysis of corrugated steel web beam bridges using spatial grid modelling. Steel \& Composite Structures, 18(4), 853-871.

57. Chen, Y.S. and Yen, B. T. (1980) Analysis of Composite Box Girders, Fritz Engineering Laboratory Library, Report N0 380.12.

58. Goel, S. C., Stojadinovic, B., and Lee, H. K. (1996) A new look at steel moment connections. Rep. No. UMCEE 96-19, The Univ. of Michigan College of Engineering.,

59. Goel, S. C., Stojadinovic, B., and Lee, H. K. (1997) Truss analogy for steel moment connections. Eng. J., Second Quarter, 43-53.

60. Lee, Kyoung-Hyeog, Subhash C. Goel, and Bozidar Stojadinovic (2000) Boundary effects in steel moment connections. Proceedings of the 12th World Conference on Earthquake Engineering.

61. Yang, Y., \& Yu, X. B. Image analyses for video-based remote structure vibration monitoring system, Frontiers of Structural and Civil Engineering, 10 (2016), 12-21.

62. Ribero D., Calçada, R., Ferreira, J., Martins, T. (2014) Non-contact measurement of the dynamic displacement of railway bridges using an advanced video-based system, Engineering Structures, 75, 164-180.

63. Leica: http://leica-geosystems.com/products/levels/leica-nivel210_220, Consulted on 31 July 2017.

64. Fleming, J. (2010) One world trade center rises, Professional Surveyor Magazine, 30, 7. 